2. There is a higher risk of skin laceration in emergency cesarean section than in vaginal delivery.

3. No vertical HCV transmission was noted among children born by elective cesarean section without skin laceration.

\section{3 'BRAIN ABSCESS IN INFANTS AND CHILDREN: A RETROSPECTIVE STUDY OF 52 PATIENTS IN PEDIATRIC'S HOSPITAL OF CASABLANCA, MOROCCO}

doi:10.1136/archdischild-2012-302724.0873

Z Jouhadi, M Lahlimi, A Rachid, J Najib. Pediatrie, CHU Casablanca, Casablanca, Morocco

Brain abscess is a serious disease of the central nerve system, it's a rare pathology in children; to review the clinical manifestation, and predisposing factors, causative organism and outcomes of brain abscess we conducted a descriptive retrospective study in children's hospital of Casablanca in unit of infectious diseases.

Fifty two infants and children with brain abscess admitted between 1998 a 2011 are retrospectively analyzed.

The mean age was 5,6 years, $38 \%$ were aged less than 2 years. the male-to-female ratio was 0,92 . The most common predisposing factor was meningitis (29\%); ORL causes (27\%); otitis (17\%) and congenital heart disease $(11,5 \%)$; the most common organisms are staphylococcus aureus; streptococcus intermedius and proteus mirabilis.

Clinical manifestation were dominated by fever (79\%); cranial hypertension $(57 \%)$; seizures $(36 \%)$ and motor deficiency in $35 \%$, sequellae were constated in 36\%; mortality in 4\%.

Brain abscesses result in significant morbidity and high fatality. Because brain abscesses can have subtle initial presentations, a high level of suspicion is very important for early diagnosis in pediatric patients, particularly those with underlying condition such as congenital heart disease.

\section{SKEWING OF SAG MEDIATED THERAPY FOR A PREDOMINANT TH1 DURING VISCERAL LEISHMANIASIS ON TRIGGERING CD2 EPITOPE}

doi:10.1136/archdischild-2012-302724.0874

${ }^{1} \mathrm{~S}$ Sinha, ${ }^{2} \mathrm{~S}$ Bimal, ${ }^{1} \mathrm{~S}$ Sundaram. 'Centre for Biotechnology, University of Allahabad, Allahabad; 'Division of Immunology, Rajendra Memorial Research Institute of Medical Sciences, Patna, India

Visceral leishmaniasis is a macrophage associated disorder which is linked with a profound decrease in the immunotherapeutic potential of the infected subjects especially children leading to a marked reduction in the CD4 linked Th1 protective immune response. Simultaneously the patients in Bihar are showing unresponsiveness towards SAG which is still a first line of drug in many countries around the world against Visceral Leishmaniasis. In the present part of the study we have tried to evaluate the use of CD2 antibody as an immunotherapeutic agent along with SAG in ensuring treatment of BALB/c mice induced with experimental Visceral leishmaniasis. It has been found in the present set of studies that stimulation of CD2 co receptor along with along with therapeutic dose of SAG has led to the enhancement in the release of IFN-gamma which leads to the release of TNF-alpha and activates the macrophages. An increase in the NO mediated killing further observed by the activated macrophages leading to the reduction in the parasitic load. The results indicate that enhancing the immune potential of a VL patient especially children will help in the better response of Sodium Antimony Gluconate which is the first line of drug against VL in many countries. LACTOBACILLI POPULATIONS FROM THE SAME HUMAN HEALTHY BIOTOPE

doi:10.1136/archdischild-2012-302724.0875
V Lakhtin, A Bajrakova, M Lakhtin, Y Belikova, S Afanasiev, V Aleshkin. G.N. Gabrichevsky Research Institute for Epidemiology \& Microbiology, Moscow, Russia

Background Probiotic bacterial lectins are important factors in biotope. The aim was to study autoregulation properties of lectins of lactobacilli (LL) isolated by us [1].

Methods Clinical strains of lactobacilli were freshly isolated from urogenital tract samples of young and adolescent patients using growth on Lactobacillus MRS Agar (HiMedia) and variant of Shaedler medium. Additional differential indication media were used. Bacterial growth was studied in the presence of disc- or dropapplied of LL on solid media or LL within bacterial suspensions (0.5-5 McFarland units). Samples studied were characterized with lactobacilli at the level of $10^{6}-10^{8} \mathrm{Cfu} / \mathrm{ml}$, and did not contain a panel of standard bacterial and viral pathogens. Decreased contents of Staphylococcus ssp., Enterococcus ssp., E. coli, and Candida ssp. were registered.

Results All methods used gave similar results (supporting each other) of action of LL on lactobacilli populations growth. The following groups of lactobacilli populations were identified: LLresistant or sensitive, weakly suppressive or stimulated, highly or moderate suppressive, highly modulated, cell condensation depended or not.

Conclusions Results indicate further evidences that probiotic LL imitate cellular probiotics. It seems LL are involved in signal events (OS, cross-talking) in biotope. LL may act as a biotope tool of microbiota selection and may be used for both supporting probiotic-like lactobacilli and simultaneous elimination of other lactobacilli possessing decreased survival (non-probiotic-like strains) in conditions of healthy or close to normal balance in biotope.

Reference [1]. V. Lakhtin, M. Lakhtin, V. Pospelova, B. Shenderov (2006) Microb. Ecol. Health \& Dis. 18: 55-60.

\section{CHARACTERIZATION OF ENTEROCOCCI CAUSING CATHETER- ASSOCIATED URINARY TRACT INFECTIONS IN CHILDREN IN IRAN}

doi:10.1136/archdischild-2012-302724.0876

'MR Pourshafie, ${ }^{2} \mathrm{M}$ Talebi, ${ }^{3} \mathrm{M}$ Saifi. ${ }^{1}$ Microbiology Department, Pasteur Institute of Iran, ${ }^{2}$ Microbiology Department, Tehran University of Medical Sciences; ${ }^{3}$ Mycobacteriology Department, Pasteur Institute of Iran, Tehran, Iran

Background and Aims Urinary tract infection is one of the most common diseases in children. Precise and untimely diagnosis and comprehensive treatment can significantly decrease late serious complications. Among microorganisms causing UTI, enterococci are one of the most prevalent reported one. Microorganisms causing UTI are resistant to most of antibiotics which lead to increasing the duration of hospitalization, morbidity, mortality and there are medical and financial implications associated with UTIs. The aim of this study was to determine enterococci prevalence, its antimicrobial resistance and its genetic diversity isolated in children.

Methods Urine samples were cultured by standard loop method. The $10^{5} \mathrm{CFU} / \mathrm{ml}$ cultures were assumed as positive. After identification of enterococci by biochemical tests, susceptibility of each isolate was assessed by disk diffusion method according to CLSI guidelines. In order to analyzing bacterial genotypic diversity, pulsed-field gel electrophoresis (PFGE) were performed using SmaI enzyme in CHEF DRIII apparatus.

Results Out of 500 urine samples, 50 were positive for vancomycin resistant Enterococci. Out of 50 VRE isolates, 3 were isolated from catheter-associated urinary tract infections from neonatal intensive care unit and Pediatric ward. All of 3 VRE isolates showed a high level vancomycin resistance $(\mathrm{MIC} \geq 128)$ and harbored vanA gene. Genotyping by PFGE using SmaI enzyme revealed the presence of two types. 